\title{
versants
}

\section{L'écriture et la vie selon Anne-Lise Grobéty}

\author{
Valérie Cossy \\ Université de Lausanne \\ ORCID: 00oo-0003-2083-5534
}

\begin{abstract}
Dix ans après la disparition de l'écrivaine, cet article s'efforce de cerner ce qui rend mémorable l'écriture d'Anne-Lise Grobéty. Sans prétendre à l'analyse rigoureuse, il invite à saisir les ressources de l'union qu'elle a tissée de sa plume entre la légèreté et la gravité.
\end{abstract}

Keywords : Anne-Lise Grobéty, écriture, langue, humour, sens, auteur, lecteur, Henri Roorda.

"J'aime bien ce mot "précieux", vous savez c'est important à l'heure où on travaille de plus en plus de façon réductrice avec notre langue. "Précieux", écoutez-moi ça comme c'est beau ! La langue est une chose précieuse.»

Anne-Lise Grobéty, Film « Plans-Fixes », 2006 (36:55-37:II).

L'œuvre d'Anne-Lise Grobéty n'a pas encore fait l'objet d'analyse érudite, mais elle est de celles dont nombre de lecteurs gardent un souvenir marquant. C'est d'ailleurs avec un engouement du public à la hauteur de l'admiration de la critique, dont témoigne Jacques Chessex dans Les Saintes Écritures, que tout avait commencé en 1969 lorsque fut décerné le Prix Georges-Nicole à Pour mourir en février, à l'unanimité d'un jury prestigieux où siégeaient aux côtés de ce dernier Corinna Bille, Jean-Pierre Monnier, Jean Cuttat, Alexandre Voisard, Nicolas Bouvier et Maurice Chappaz. Aujourd'hui, plus de dix ans ont passé depuis la disparition prématurée de l'écrivaine et, sans être prête à fournir une analyse digne du nom, j'aimerais contribuer, en prof de littérature dont c'est le métier, à garder la flamme en vie pendant la traversée du désert à laquelle s'apparente parfois l'immédiate postérité d'une œuvre jusqu'à ce que les jeunes générations s'en emparent.

Deux impressions contradictoires s'imposent lorsque nous relisons Anne-Lise Grobéty ou écoutons ses propos - qu'il s'agisse de la longue interview par Charles Sigel dans le film «Plans-Fixes » en 2006 ou de celle, plus ancienne, de l'émission La Voix au chapitre (TSR 1973), dont Jérôme Meizoz nous a récemment rappelé l'existence (Meizoz 2020 : 29-32). L'écriture est à la fois une expérience et un accomplissement dont Anne-Lise Grobéty

I Anne-Lise Grobéty en dresse scrupuleusement la liste dans le film «Plans-Fixes » (06:4007:02). 
parle avec précision et spontanéité et, en même temps, l'écriture n'a pas l'air d'avoir toujours occupé le centre de sa vie, au point qu'il nous arrive de douter si, derrière sa visible désinvolture, elle-même avait pris la pleine mesure de son talent. Mais comme l'indique sa manière de poser la question en I983, elle a toujours tenu à souligner qu'indépendamment même des aléas propres à l'existence d'une femme suisse bien occupée de sa génération, l'écriture et la vie sont fondamentalement incompatibles. Interroger le prix à consentir pour éventuellement en arriver à sacrifier l'une pour l'autre correspondait à sa façon d'envisager le problème : "peut-on faire à la fois deux choses aussi importantes que vivre et écrire? [...] on vit forcément aux dépens de l'écriture, on écrit forcément aux dépens de la vie... ", disait-elle (Grobéty I983: 8). Son œuvre pose donc, comme première brique, cette exigence de la présence au monde sans laquelle une vie humaine ne vaut d'être vécue. En être privée, tel est potentiellement le prix exorbitant de l'écriture: écrire a donc intérêt à en valoir la peine ! Et parfois, elle l'admettait aussi, Anne-Lise Grobéty n'avait tout simplement pas pu faire autrement. Qu'elle l'ait ou non désiré, écrire faisait intimement partie d'elle et de sa façon d'être au monde, la vie et l'écriture s'imbriquant de manière plus ou moins harmonieuse, mais forcément nécessaire dans le tout-venant des jours.

À l'automne 2009, alors que la maladie qui allait l'emporter venait de se faire sentir pour la première fois, elle avait tenu à mener presque comme si de rien n'était la réalisation du dossier que la revue viceversa était sur le point de lui consacrer et dont j'avais eu la chance d'être chargée. Ayant dû renoncer à nous rencontrer chez elle, nous nous étions rabattues sur l'écrit et l'échange virtuel : je lui avais envoyé par courriel une liste de questions auxquelles elle avait répondu en détail. Vu la taille finale de l'entretien écrit, une partie seulement de ses réponses purent prendre place dans le numéro 4 de la revue viceversa en avril 20I0, tandis que l'entier de son document Word repose encore sur mon disque dur, m'obligeant à me poser la question qui était la sienne : cette trace écrite magnifique, ne l'ai-je pas acquise aux dépens de sa vie? Ce qui est certain, c'est que mes notes prises autour d'une tasse de café auraient été infiniment plus pauvres.

\section{Ce qu'écrire veut dire}

C'est donc en suivant ce qu'elle-même disait de sa conception de l'œuvre et de son rôle d'auteure que je souhaiterais entamer la réflexion : "J'aime bien me sentir en tant qu'auteure comme la passagère clandestine du texte en train de se faire » (viceversa 4 : IO4). Par cette formule, Grobéty exprimait la manière dont elle est toujours présente car l'auteure qu'elle est ne peut jamais complètement s'absenter, fût-ce d'un texte de fiction. Son écriture se joue à la frontière entre l'expérience singulière et l'imaginaire aux origines pas 
toujours connues, entre le soi de l'écrivaine et l'expérience collective : «J'ai besoin de rester présente, en alerte, en arrière-plan, dans une sorte de jeu sur le fil entre la peau de la narratrice, par exemple, et la mienne » (IO4). Dans Compost blues elle définissait l'objet-livre tiré de la matière première $\mathrm{du}$ bois des arbres comme une « texture d'échanges en profondeur entre vie réelle et réalité de la vie écrite " (Grobéty 2000 : 9) : pas d'opposition ni de contraste en noir et blanc, selon elle, entre la réalité et la représentation, mais une circularité voire un recyclage sans fin dont on ne sait plus où il a commencé, qui nous fait passer d'une vie à une autre. L'écriture se déploie, sous sa plume à l'encre souvent turquoise, dans un rapport d'échange avec la vie ordinaire, avec un dialogue qui commence en général par s'imposer à elle sans crier gare. Répondre à de telles injonctions intimes à l'écriture doit rendre possible, au final, une compréhension élargie voire inattendue de la réalité communément perçue. Un peu plus loin, en réponse à la même question, elle précisait :

J'exerce constamment en même temps cette double responsabilité - celle de l'écrivaine au travail et celle des personnages en exil dans l'histoire... La refonte du réel en fiction (Gide parlait de "refondement »), en texte littéraire, exige une constante vigilance, une intelligence dans son sens premier pour relier le particulier d'une histoire à l'immense caisse de résonnance du monde et des blessures communes. C'est ce que je ne voudrais jamais perdre de vue quand j'écris (viceversa 4 : I04).

Ce lien indissoluble entre la vie de tous et son écriture singulière prenait au fil de ses propos la forme d'un triangle qui la reliait à ses lecteurs, que ce soit dans le film «Plans-Fixes » ou, ici, dans le même passage de l'entretien, lorsqu'elle définit son travail d'écrivaine :

De toute façon, c'est la conjonction de trois mouvements, un travail en triangle - de l'auteur aux personnages, de l'auteur aux lecteurs par ces réflexions évasées et du lecteur au texte - qui donne sa texture à l'écrit (IO4).

Il était clair pour elle que le texte échappait à son auteur une fois remis entre les mains des lecteurs... mais jamais complètement. Elle qualifiait aussi l'écriture de travail de « refonte » ou de « refondement de la réalité » car elle impliquait à ses yeux la possibilité et même le devoir d'en faire bouger les lignes de représentation afin de déstabiliser les consensus conventionnels sur ce qui semble aller de soi, aussi solides fussent-ils. C'est comme ça qu'elle envisageait notamment le roman : « la fiction, c'est justement d'aller se poser à l'extrême bord, là où se brisent les certitudes » (partie inédite de l'entretien). Une autre expression qui revenait régulièrement chez elle pour qualifier son travail artisanal d'écrivaine était le mot "évasement" (cf. ici 
ses « réflexions évasées »). Sous ce terme elle désignait le travail opéré par l'imaginaire de l'auteure et son écriture à partir du point fixe et resserré de la réalité perçue par le sens commun, elle-même devant dépasser le point d'amarrage, en quelque sorte : "évasement" signifiait alors que cet élargissement ne pouvait se faire qu'à partir d'une expérience vécue banale, celle des contraintes et des conventions, qui peuvent correspondre à celles des lecteurs, dont l'écrivaine élargit par son travail les ressources d'interprétation en leur donnant accès à d'autres façons de lire le monde.

Le rôle de l'écrivaine vis-à-vis de ses lecteurs consistait selon elle à stimuler leur autonomie et leur confiance en "déverrouillant" - autre terme cher à Grobéty - leur propre intelligence critique et leur propre créativité dans leur rapport au monde :

Je n'ai rien à imposer à mes lecteurs, sinon leur proposer sous l'histoire qui court des choses tirées de la vie et de ses ambivalences, je les veux pleinement recréateurs et capables de mettre en route leur subjectivité jusque dans le texte. En espérant qu'ils auront envie d'avancer vers un peu plus d'autonomie, de liberté, en projetant leurs propres interrogations à travers certains éléments du récit (partie inédite de l'entretien).

À leur propos, elle disait aussi : «J'exige beaucoup d'eux, je le sais, mais j'ai en retour pleinement confiance en eux ! (partie inédite de l'entretien). Elle envisageait la littérature comme une affaire de collaboration entre une auteure et des lecteurs tout sauf passifs : comme une aventure commune au sein de laquelle son écriture déployait de nouvelles façons de voir dont ceuxci devaient s'emparer à leur tour.

\section{Écrire le réel insaisissable}

Dans une revue consacrée à Henri Roorda, l'on ne saurait ébaucher une analyse de l'œuvre d'Anne-Lise Grobéty sans évoquer ses Contes-Gouttes (I986) dont l'humour et l'absurde entrent en résonnance avec les chroniques du fantasque professeur de mathématiques. Elle-même a aiguisé la curiosité de ses lecteurs présents et à venir en admettant à propos de son rapport à l'humour:

le ton des Contes-Gouttes est typiquement quelque chose qui ne peut s'imposer que sur des textes très courts, vifs et remuants. Ce qui est curieux, c'est que j'y ai donné ma vision du monde comme dans aucun autre ouvrage, je crois que ce sont les textes les plus engagés que j'aie écrits, mais je n'ai pu le faire qu'à travers ce ton qui paraît désinvolte, où les mots sont sans cesse en perte d'équilibre (viceversa 4 : Io9). 
Première conclusion : il faut se méfier de la désinvolture d'Anne-Lise Grobéty. Mais comment, ensuite, tirer des Contes-Gouttes une vision du monde ? Voilà une question qui aura de quoi occuper étudiantes et étudiants pendant les années à venir tout en déstabilisant durablement leurs professeurs... Bravo Anne-Lise !

Faisons donc le pari de prendre au sérieux l'Arpenteuse de nuages, l'Avaleuse de couleuvres, la Dame-écho et autres Inspecteur de parapluies, Brasseur de vide ou Guetteur d'oiseaux, ces créatures irrépressiblement nées des longues séances passées au Grand Conseil neuchâtelois où siégea Anne-Lise Grobéty pendant dix ans... car sa vie ne fut jamais synonyme de contemplation seulement mais aussi d'engagement politique et de participation active au cœur des échanges et affrontements humains. Alors que l'on fête aux quatre coins du pays le cinquantenaire du suffrage féminin acquis au niveau fédéral le 7 février 1971, il est bon de se souvenir qu'elle tint sa place dans le législatif de son canton à une époque où l'engagement des femmes en politique était encore toute une affaire ${ }^{2}$ ! Elle participa concrètement à l'élaboration des lois ainsi qu'aux très longs et parfois ennuyeux débats que requièrent les compromis qui les font aboutir. Et c'est de là que surgit l'écriture des Contes-Gouttes :

Être au Parlement, c'est pas passionnant à chaque minute. [...] J'ai trouvé là, au fond, l'idée du conte-gouttes, c'est-à-dire cet étage de jeux avec les mots, en entendant un peu certaines sornettes qui se racontaient, avec la difficulté, un peu, de ne pas pouvoir exister avec son propre langage : cette frustration je l'ai déjouée en commençant à écrire ces contes-gouttes (« Plans-Fixes » : 36:00-36:27).

Le parlement fut ainsi le moment de vérité de l'écrivaine : il y a langue et langue. Les lois sont nécessaires, mais leur langue n’est pas la sienne ni celle de la littérature. Les premières visent à être les moins équivoques possibles en débarrassant la langue, en fait, de ses ambiguïtés. Or celles-ci constituent ce qu'elle appelle l'« énergie renouvelable » des mots de l'écrivain, expression par laquelle elle qualifie régulièrement les ressources par définition inépuisables de la langue littéraire. Répondant à une question de Charles Sigel sur son goût atypique de la lecture dans le milieu ouvrier qui était le sien, elle évoque pour l'expliquer « une forme de confiance dans les mots » : « Je savais qu'ils m'apportaient quelque chose du monde, de l'extérieur. » Ils lui ont donné, dit-elle, «ce sentiment de l'énergie prodigieuse

2 Neuchâtel fut le deuxième canton suisse après Vaud à accepter par $54 \%$ des voix, lors de la votation populaire (masculine) du 27 septembre 1959, de reconnaître aux femmes leur condition de citoyennes à part entière en leur octroyant le droit de vote; La Chaux-de-Fonds, où grandit Anne-Lise Grobéty qui avait alors dix ans, fut la ville la plus généreuse, avec $67 \%$ de "oui". 
de la langue à notre disposition et, miracle, c'est une énergie renouvelable » (05:09-06:23). L'écriture de Grobéty ne saurait s'appuyer sur un sens parfaitement circonscrit des mots, tel qu'exigé par l'élaboration des lois. Son écriture est éminemment dépendante, au contraire, de leur instabilité, de leurs glissements, de leurs zones d'incertitude qui ouvrent à la subjectivité en alerte de nouvelles perspectives. L'écriture qu'elle pratique, qui la rendit parfois impatiente ou déconcentrée au Grand Conseil, est celle qui ne prend aucun mot pour acquis, mais préfère jouer avec eux afin de conférer aux lecteurs le pouvoir de deviner leur mode opératoire arbitraire : la force qu'ils exercent prosaïquement au quotidien en imposant une certaine forme au réel que l'écrivaine va nous faire voir autrement.

Les faux étonnements de Henri Roorda anticipent souvent le ton pseudo-naïf des Contes-Gouttes. Comme ceux-ci, les chroniques du professeur-journaliste nous invitent à nous amuser du caractère irréductible du réel, tandis que ceux qui font les lois croient être en mesure de le maîtriser : « la parfaite sagesse du Législatif ne suffit pas à mettre un ordre durable dans les choses ", feint de regretter Balthasar (Roorda 20I3 : I2I). À l'image des échecs qu'auront à subir les Tourneurs de pages de Grobéty, les «saisons indisciplinées » de Roorda ne sauraient se conformer à la régularité envisagée par leur Régisseur pour organiser le monde équitablement de manière prévisible : «Jamais, pas une seule fois depuis que les humains contemplent le spectacle, les Saisons ne sont entrées en scène à l'heure exacte " (I2I). Comme la narratrice des Contes-Gouttes, le chroniqueur joue de l'absurdité humaine consistant à voir partout des liens de cause à effet en confondant les mots et les choses. Cette façon de mettre à nu l'arbitraire et la prétention des faiseurs de sens tout en s'amusant de la dimension indomptable du réel est peut-être ce que l'on pourrait qualifier, chez Grobéty, de vision du monde : nier par avance toute possibilité raisonnable de contenir le monde par le sens et savoir trouver, en tant qu'écrivaine, la juste distance vis-à-vis de la volonté humaine de le réduire en signes. Peut-être s'agit-il là d'une façon pour nous de comprendre le "sens" de ses «Tourneurs de pages » : « Il était une fois des tourneurs de pages. Leur tâche consistait à tourner, juste au bon moment, les pages de l'Histoire et celles de la vie » (Grobéty 1986 : II3). Inutile de dire que la tâche de ceux-ci est tout autant irréalisable que celle du Régisseur de Roorda : «Il était encore moins facile dans ce domaine de savoir s'il était exactement ni trop tôt ni trop tard » (II7). En intervertissant allègrement, voire en troublant complètement, l'ordre du discours que l'on croyait maîtriser entre les choses et les signes, Grobéty, comme Roorda l'a fait en son temps, nous invite à regarder à distance les explications raisonnables que l'on croit solides et définitives. Comme le dit ce dernier : « Si les savants ne croyaient pas en la simplicité des lois de la nature, ils abandonneraient leurs laboratoires et leurs livres pour se livrer à la boisson. On 
peut heureusement faire croire aux hommes tout ce qu'on veut " (Roorda 2013: I2I). Mais, si Grobéty partage une telle critique des visions explicatives qui se croient supérieures, les prétentions et même la bêtise humaine ne la rendent pourtant pas tout à fait aussi pessimiste. Tout de cette humanité - certes faillible de génération en génération - n'est pas à jeter. Celle-ci est même bien aimable tant que l'on peut rire des défauts que "nous" avons en partage :

Donc, le brasseur de vide de notre enfance va disparaittre à son tour. C'est la rançon du progrès. Et nous serions malvenus de faire de la sensiblerie en nous raccrochant au "bon vieux temps". Brasser le vide réclame des forces nouvelles (Grobéty 1986 : I08).

$\mathrm{Si}$, mise en garde par l'écriture de Roorda et Grobéty, je refuse de m'aventurer à pérorer sur le sens de l'œuvre de la romancière ou à diagnostiquer ce que pourrait être sa vision du monde, je pense néanmoins que les ContesGouttes, au-delà de leur trompeuse simplicité, ont de quoi nous révéler quelque chose d'assez fondamental sur l'auteure Anne-Lise Grobéty : ils mettent en lumière sa façon de toujours construire et déconstruire en même temps, le rapport à la langue étant précisément vécu par elle comme un jeu de construction. Se livrer à l'exercice supérieur consistant à élaborer du sens, quel que soit le registre dans lequel on se déploie, ne doit jamais faire oublier les pièces élémentaires - building blocks, disent les Anglais - dont il est constitué. Voici, par exemple, comment Grobéty décrit, sur le mode du récit d'origine de la littérature, sa toute première rencontre avec la langue écrite au fil du b-a-ba du livre de lecture de l'école primaire - « médor mord le chat [...] mimi a un chat »:

Ce sont ces ressassements, ces piétinements de si peu d'importance pour l'homme ou pour l'humanité qui vont finir par nous mener à Victor Hugo, Proust ou Rimbaud (arthur) ou aux romanciers américains contemporains ou même à Lacan.

C'est parfois

Sur une poussée

de toutes petites vérités

que prennent souche ou pied

nos plus grandes évidences (Grobéty 2000 : 13).

Le ridicule et le sérieux, le grand et le dérisoire sont indissociables et se nourrissent l'un de l'autre, ne cesse-t-elle de nous dire, c'est peut-être ça être humain. La démystification des hiérarchies, même lorsqu'elles sont nécessaires, est une constante de son écriture. Son poème évoque, mine de rien, 
Emily Dickinson et la manière qu'a celle-ci, pour dire la vie humaine, de faire se côtoyer contre nature mais comme une évidence le très concret et le très transcendant : « Tell all the truth but tell it slant [...] / The Truth must dazzle gradually / Or every man be blind - » (Dickinson \# I263).

\section{Toujours écrire et toujours en vie}

La division entre le drôle et le triste, entre la comédie et la tragédie, est régulièrement mise en évidence par Grobéty comme un arbitraire formel, dont la binarité ne convient pas à son écriture. Elle reconnaissait pourtant - et l'aveu peut surprendre ses lecteurs - que chez elle dominait la gravité. À un niveau purement biographique, elle révèle dans l'entretien avec Charles Sigel qu'au moment de son premier roman (qui se termine quand même sur une note légère), elle était en fait "quelqu'un de très triste ", ajoutant que ses enfants, loin d'être seulement celles qui ont empiété sur le temps de l'écriture, lui ont carrément permis de voir la vie sous un autre jour : "Ces trois filles, elles m’ont aidée à ne pas rester dans la pente de la gravité » («Plans-Fixes» :46:00-46:20). L'expérience procure ainsi des changements de tonalité que l'écriture à elle seule ne saurait accomplir, et elle tenait à le dire. Alors que je l'interrogeais en 2009 sur la cohabitation entre souffrance et pulsion comique chez ses personnages de narratrices, elle répondit :

Tout simplement, je vis comme ça, dans le refus de la tragédie, de l'emphase [...]. Le rire, l'humour, la distanciation est la plus sûre valeur pour ne pas rester en plan, tout comme la poésie composante intégrale de la vie. Humour, amour et poésie sont toutes trois de formidables réserves d'énergie renouvelable [...]. Bien sûr, quand j'écris je me tiens d'abord dans la pente de la gravité. Mais, justement, être grave n'empêche ni la douceur ni la gaîté. On est là en pleine musique baroque, non ? (partie inédite de l'entretien).

Avec son grand roman posthume, Des nouvelles de la Mort et de ses petits, qu'elle n'a pas pu présenter elle-même à ses lecteurs, Grobéty avait fait le pari d'aborder le sujet le plus univoquement tragique - la Mort - sur un mode baroque, comme le suggère la photo de couverture de l'édition originale parue chez Campiche, qui illustre la scène du théâtre du château de Cesky Krumlov dans la République tchèque. L'association entre tournure comique, longueur du roman et sujet du texte constituait un exercice de style inédit voire un défi pour l'écrivaine, qui confiait mystérieusement en 2009, à propos de l'humour dont ce dernier roman, à l'image des ContesGouttes, serait investi : 
Disons qu'il y a des tonalités qu'il est plus difficile de conserver sur de longues distances sans risque de se casser la figure. Encore que l'expérience que je suis en train de mener avec mon prochain roman pourrait me contredire... (viceversa $4:$ Io9).

Un extrait du « roman en travail » était publié dans la revue, en préambule à l'entretien : on pouvait y découvrir une écriture romanesque tentant d'apprivoiser le drame absolu de la mort d'un enfant grâce au registre de la distanciation (98-Ioo). L'humour sur la longue distance de cet audacieux roman expérimental attend aujourd'hui des analyses et des commentaires que je ne suis pas en mesure de livrer ici.

Mais, en même temps qu'elle en écrivait les dernières pages et répondait à mes questions, Anne-Lise Grobéty venait d'apprendre qu'elle allait devoir affronter l'épreuve de la maladie, sans savoir laquelle des deux finirait par emporter la bataille. L'on pourrait penser que toute possibilité de "distanciation" et donc d'écriture devenait, dans de telles circonstances, tout bonnement impensable. Certes, écrire à partir de l'expérience de l'épreuve, Anne-Lise Grobéty connaissait. Lorsqu'elle évoquait, par exemple, les affres de Laurence dans Zéro positif (1975) et la forme d'"écriture féminine" qu'elle avait déployée pour en parler, elle disait : «Je n’ai, en fait, jamais travaillé autrement qu'en étant concentrée sur cette forme de nécessité intérieure » (I07). Ou, pensant à son dernier roman encore inachevé, elle disait aussi :

Ce qui me paraît essentiel, une fois de plus, comme dans les romans, c'est que même dans les pires moments rien n'est jamais complètement désespéré, c'est toujours la vie, la vitalité, qui a le dessus. Pour mes personnages, l'écriture peut certes avoir valeur de réparation, mais je crois d'abord que, dans le creusement des phrases, se cache l'expression de la volonté de réparer l'autre, de prendre soin du monde et de ses vivants. Le rire, l'humour, la pirouette en sont les meilleurs soignants (I09).

Même à ce moment qu'elle savait déjà lourd d'incertitudes, elle choisissait d'écrire que "rien n'est jamais complètement désespéré », et elle utilisait le mot "pirouette". Dans la partie demeurée inédite de l'entretien, elle disait aussi : " Je n'avance en écriture que grâce à l'incertitude, au doute, à tout ce que je ne sais pas de ce qui va se passer» (partie inédite de l'entretien).

En 20I0, Anne-Lise Grobéty écrivit son dernier texte en se doutant qu'il pourrait être le dernier : un poème en prose, Bruit blanc Bruit noir, surgi de ce qu'elle ne savait pas de ce qui allait se passer. L'écrivaine regardait la mort - sa mort - et en tirait ses mots pour décrire cette rencontre qui nous concerne tous. "You Want It Darker ", se plaint Leonard Cohen auprès d'un Dieu ignorant la clarté des millions de bougies «burning / For the love that never came ». Comme lui Grobéty pourrait paraître à première vue hésiter entre 
deux alternatives, comme si la mort réduisait à deux la complexité de la vie : noir-blanc, l'obscurité ou la flamme. Comme lui, certes, elle nous confronte au désespoir. Mais pourtant, elle nous offre aussi la nuance qui continue d'exister, elle le sait, hors de sa subjectivité assiégée.

Le bruit noir ou blanc, l'alternative du dedans ou du dehors, de l'avant et de l'après, du quelque chose et du rien certes hantent la vie intérieure de la narratrice sur un mode forcément terrible et tranché. Mais elle n'en oublie pas moins la vie extérieure et ses nuances : «Les bleutés, le grisé, à moins que ce soit le blanc qui a juste un peu vieilli » (Grobéty $2010: 4$ ). Elle nous fait partager son contact avec la montagne. "Avancer en écriture", l'expression prend tout son sens dans ce texte qui commence avec le verbe "partir" et au fil duquel la trajectoire de l'écrivaine se confond avec la trajectoire de l'alpiniste : «On sent l'orgueil de tracer sa route au bord de la nuée » (5). Mais la blancheur des sommets se confond malheureusement avec la lumière des néons de l'hôpital et, tandis que les déplacements des rochers et des animaux s'accompagnent des sons de leurs craquements et de leurs cris, la révolution qui affecte la nature en microcosme du corps de la narratrice s'est faite, nous dit-elle, sans bruit, «dans le plus pur silence au fond de soi » (I5). La fin de ce poème en prose est - hélas ! pour tous ceux qui aimaient Anne-Lise - prémonitoire : pour elle comme pour le sérac menacé de chute. Nous qui sommes ses lecteurs demeurons habités par l'ultime cadeau laissé par son écriture.

Et nous restons chargés de la responsabilité de faire vivre avec ses textes leur passagère clandestine, qu'était toujours Anne-Lise Grobéty :

En fin de compte, c'est toujours l'œil du lecteur qui met le texte au monde.

Ce texte vivant, certes, mais recroquevillé dans sa poche d'air et dans le noir, sachant que seul le regard démultiplié de l'autre va le dé-livrer et le ramener à la lumière.

Ce qui joue alors en faveur de ses heures de survie ?...

Coincé pour toujours dans la boîte fermée du livre, redeviendra poussière

ou cendres

ou terreau

- nul ne peut le dire (Grobéty 2000 : 19). 


\section{Bibliographie}

"Anne-Lise Grobéty », Viceversa littérature, nº 4, avril 20I0, pp. 96-II3.

Chessex, Jacques, Les Saintes Écritures, Vevey, Bertil Galland, 1972.

Cohen, Leonard, You Want It Darker, Nashville, Sony (music), Old Ideas LLC (texts, images and photographs), 2016.

Cossy, Valérie, «Entretien inédit avec Anne-Lise Grobéty en vue du dossier de la revue viceversa $\mathrm{n}^{\circ} 4$ ", document Word, octobre 2009.

Dickinson, Emily, « Poem I263", The Norton Anthology of American Literature, Seventh edition, vol. C, I865-19I4, London and New York, Norton, 2007, p. 9 I.

Grobéty, Anne-Lise, « Du côté de l'écriture féminine », dans Anne-Lise Grobéty, Monique Laederach et Amélie Plume, Littérature féminine ou féministe ?, Carouge-Genève, Zoé, 1983, pp. 6-27.

-. Pour mourir en février - Zéro Positif - La Fiancée d'Hiver, Lausanne, 24 heures, 1984 .

-. Contes-Gouttes, La Tour-de-Peilz, Bernard Campiche, 1986.

-. Compost blues, à sept temps plus ou moins décomposés, Lausanne, A[ch]LLfAssociation Suisse des Libraires de Langue Française, 2000.

-. Bruit blanc Bruit noir (avec une pointe sèche de Francine Simonin), Lausanne, Bibliothèque cantonale et universitaire, 2010.

-.Des nouvelles de la Mort et de ses petits, Orbe, Bernard Campiche, 20II.

Meizoz, Jérôme, Faire l'auteur en régime néo-libéral. Rudiments de marketing littéraire, Genève, Slatkine Érudition, 2020.

Roorda, Henri, Les Saisons indisciplinées, éd. Gilles Losseroy, Doris Jakubec et Carine Corajoud, Paris, Allia, 2013.

Sigel, Charles, "Anne-Lise Grobéty », Association Films " Plans-Fixes », 2006, https://www.plansfixes.ch/films/anne-lise-grobety/ 